\title{
Intervención psicosocial, redes sociales y estilos de vida. Conceptos de referencia y abordaje en la salud mental del país
}

\author{
Domingo Asún S.*
}

\begin{abstract}
The purpose of this article is to describe and characterize the present and future relationship between Social applied psychology and both political and social programs fields. Assigning to the mental health area as a central part of this relationship, macro and microsocial factors are analized in the socio-historic context of the changes occurred in the country' and its impact in both subjetivity and life quality process. Since the focus of psychosocial nets intervention, take councieuss of this proposal, its racionality, fundamentation and perspectives. From of both cultural and sociopolitical context derivative aspects and actor's character of social process in ambients both disfavoring and without power it suggests to enfathize a perspective's change to improve the ethics validity, the implications and the real contribution of this focus.
\end{abstract}

\section{Resumen}

Este articulo tiene como objetivo describir y caracterizar la relación actual y futura de la Psicología social aplicada con el campo de las políticas y' programas sociales. Destacando como dimensión central de esta relación el área de la salud mental, se analizan los factores macrosociales y microsociales en el contexto sociohistórico de cambios que ocurren en el país y su impacto en procesos de subjetividad y calidad de vida. Desde el enfoque de la intervención psicosocial en redes, se da cuenta de esta propuesta, su racionalidad, fundamentos y orientaciones. A partir de los aspectos derivados del contexto cultural y sociopolítico y del carácter de los actores de procesos sociales en ambientes desfavorecidos y deprivados de poder se propone destacar un cambio en las orientaciones para mejorar la validez ética, las significaciones y el aporte real de este enfoque.

- Psicólogo Social/Universídad Diego Ponales
Antecedentes

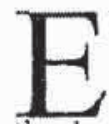
n Chile, en los últimos años, se ha hecho evidente una participación creciente de la psicología social, como disciplina de las ciencias sociales en los programas destinados a mejorar la calidad de vida y el bienestar subjetivo de la población (1). Esta situación es aún emergente, con altibajos, tiene fragilidad, porque pese a lo agudo y dramático de algunos problemas, las relaciones entre la psicologia social y comunitaria con las políticas sociales es débil y carece de visiones y recursos institucionales que permitan avances y consolidación (2). Sin embargo, todo parece señalar que la psicología social puede aportar teórica y metodológicamente en el análisis y elaboración de proyectos de intervención. Un análisis socio-historico de la investigación psicosocial en Chile entre los años 1975 y 1996 realizada por Asún, Paéz, Vera y colaboradores da cuenta de que el papel de la psicología social se hace cada vez más evidente en los programas de desarrollo social (3).

Esta situación parece particularmente relevante en el ámbito de la salud mental. Chile aparece como un mundo cambiante y dinámico, pleno de incertidumbre y acontecimientos difícilmente predecibles en el que los problemas que afectan a la población representan un reto para los científicos sociales.

\section{La necesidad de dar prioridad a la salud mental}

Diversos estudios dan cuenta que hoy es mayor la cantidad de individuos y grupos que se perciben en situación de malestar y desventaja (4). Sentirse solo, aislado, desalentado, abrumado, etc., es comparativamente, en términos internacionales e históricos, una situación frecuente. Muchas personas viven con problemas acuciantes y además en extrema tensión para lograr su supervivencia. Esto afecta su desarrollo personal, su imagen de si, su proyecto de futuro, sus relaciones sociales y sus vínculos 
primarios. Estar en situación permanente de esfuerzo máximo, aceleramiento extremo y esforzado por encontrar solución a problemas es la caracteristica psicosocial, casi más presente en el ciudadano, con que llegamos al siglo XXI. El estado colectivo de la salud mental se afccta por los problemas de empleo precario y cesantia, un mercado laboral arbitrario y autoritario, cambios vertiginosos y sentimientos de pérdida de control en las transformaciones sociales, culturales, tecnológicas y políticas.

En nuestro país, problemas macrosociales tan diversos como los originados por los cambios ideológicos, económicos, políti$\cos$ y culturaies acaecidos en las últimas décadas han tenido un fuerte impacto social, que se ha reflejado en estilos de vida cada vez más individualistas; el exacerbado consumismo; el reto de afrontar fisuras y conflictos en la población más desfavorecida y problemas sin aparente solución en el área de promoción de la salud, como el Sida, las drogas y la contaminación ambiental, entre otros. Sin duda, éstos representan necesidades y demandas sociales que requieren de un análisis científico riguroso y de respuestas consonantes para ello.

En este marco, los problemas sociales más frecuentes que afectan tanto la calidad de vida y el bienestar psicosocial, además de agravar desequilibrios en injusticias sociales, a modo de ejemplo, se encuentran por un lado, aquellos producidos por el abuso y consumo de drogas y otras sustancias (5) los cuales a su vez, están intimamente vinculados a la delincuencia y victimización (6) y, por otro, aquellos más presentes en poblaciones de alto riesgo como violencia intrafamiliar y maltrato infantil (7) inadaptaciones $y$ fracasos escolares (8) y todos aquellos problemas relacionados con la salud mental y psicológica y que el Minsal y los scrvicios de salud han planteado en el último tiempo.

En este sentido, debemos también considerar aquellos problemas relacionados con el bienestar social y psicológico producidos por estresores sociales como las desigualdades en la distribución de la riqueza, crisis económicas y el pobre mercado laboral, y que tienen profundos efectos en la familia, la paternidad y en última instancia en el ajuste psicosocial de niños, jóvenes, adultos y ancianos (9).

En nuestro pais los índices macroeconómicos indican que la economía nacional ha asumido un curso favorable; signo de estabilidad. Sin embargo, los efectos positivos de este progreso no se están distribuyendo equitativamente entre los diferentes sectores de la población. Al parecer sucede exactamente lo contrario. Se está potenciando la desigualdad y paralelamente o como contrapartida, una mayor demanda de recursos para satisfacer necesidades de supervivencia cada vez más acuciantes, en los sectores más pobres de la sociedad. Algunos datos, a modo de ejemplo, dan cuenta de que existe un $35 \%$ de desempleo juvenil en los sectores más pobres (Encuesta CASEN, 1990), el aumento de la desigualdad en el reparto de la renta y de la riqueza (PNUD, 1996), en las diferencias en el nivel educativo, en los problemas en torno al hacinamiento - allegados - y a la calidad residencial y vivienda y en la atención primaria de salud. Esto hace que las desigualdades y problemas sociales como la marginación social y la mayor conflictividad en los sectores de extrema pobreza, se agudicen. Es esto lo que se expresa en problemas como la drogodependencia, delincuencia, desempleo, subempleo, etc., y que en realidad para muchos son el sintoma y excepcionalmente el verdadero problema.

La respuesta a lo antes señalado, ha sido, por lo general, de tipo sociopolitica y consiste en atender fundamentalmente a los datos macrosociales, arbitrando medidas socioeconómicas para paliar tal estado de cosas. Desde este enfoque, se apuesta por una intervención estatal en los problemas sociales a través de la planificación de políticas sociales y se considera como la mejor soluciỏn un incremento en la inversión en el área de los servicios sociales. La finalidad es corregir en la medida de lo posible las desigualdades existentes, lo cual aunque positivo no es suficiente.

Desde nuestro punto de vista, las orientaciones teóricas que dan contenido y sentido al estudio de las estrategias de acción social con la finalidad de satisfacer las necesidades sociales mencionadas, son variadas, diversas y a veces poco rigurosas, dejando al margen muchas veces los reales aportes de las ciencias sociales.

Finalmente, esto termina expresándose en que, por un lado, se demanda que las políticas de salud mental se preocupen de modo preferente por la calidad de vida y el bienestar de tocos y que en la base social aumentan, con diversas expresiones culturales y sociales, las demandas de apoyo efectivo, social y de seguridad.

Expresados en cifras, estos problemas relacionados a la salud mental y psicológica, muestran una prevalencia desalentadora. La desigualdad social creciente y el aumento enajenado de los tipos y expectativas de consumo son los estresores sociales que hacen de telón de fondo de los sintomas.

La psicologia social aparece, en este panorama, como una dimensión privilegiada para facilitar procesos de cambio, de mejor utilización de recursos, de potenciación de identidades, de aplicaciỏn de estrategias.

No hacer uso de su potencial es un grave riesgo y también lo es, hacer uso incorrecto, parcial y reduccionista de sus posibilidades. Algunas aplicaciones contemporáneas en nuestra sociedad, se han referido a aspectos psicosociales; dentro de ellas el uso de la intervención psicosocial de redes para optimizar recursos y mejores estilos de vida. 


\section{Redes e intervención psicosocial}

Todo lo anterior hace urgente ia tarea de reflexionar y revisar qué se ha hecho, pues, una aplicación en el vacio social puede constituirse en el más desafortunado paso para castrar y frustrar las posibilidades productivas y humanizadoras, tanto en la acción social transformadora como en la investigación, que esta perspectiva auto-reflexiva y organizada lucha constantemente por plantear (10).

Inquietud fundamental de esta visión ha sido demostrar como aspectos estructurales: que la injusticia social y el desempleo influyen en el desarrollo de síntomas psiquiátricos. En esta relación aparece la mediación de factores como pérdida de apoyo social, soporte afectivo y fisuras en la autoestima. En Chile, ya hay estudios que verifican que el apoyo social y la integración a redes sociales actua como situación de protección contra estresores y facilita mejor bienestar psicológico e incluso salud física (3).

A partir de esto, la relación de redes sociales e intervención, ha desatado un verdadero delirio nacional donde la verborrea no deja ver el bosque. Se ha generado una avalancha de capacitaciones, órdenes, textos, documentos, seminarios, licitaciones con intencionalidad psicosocial y con metas estratégicas en instalación de redes. A veces pareciera que dimos con la piedra filosofal, si no en las políticas sociales, al menos en las de salud mental y calidad intersubjetiva de vida.

Es necesario poner un cable a tierra. Se ha ordenado formar redes para cualquier asunto: mujer y género, maltrato infantil, infancia en riesgo, violencia intra e inter familiar, abuso sexual, prevención de drogas.

A nivel de instituciones: diversos sectores, reformadores sociales, educadores sociales, usuarios etc., la adoptan como discurso, superando el interés por la "Autoestima y Talleres" de los años ' 80 . Se plantean con cualquier cobertura: locales, comunales, regionales. La exageración ha llevado al talismán, hasta el interior de los servicios públicos, lo cual es, a lo menos, paradójico; es hacer aparecer a la prehistoria como modernidad. Organizarse para repartir recursos no es ninguna innovación.

La reflexión critica nos señala que la red social indica un crecimiento de la acción organizada, una recuperación del tejido social fragmentado, una superación de quiebres y rupturas. En definitiva, puede ser una buena idea para programas de orientación humana-ecológica que facilite y promueva la modificación exitosa de aspectos negativos del ambiente, que disminuya aspectos de complejidad dificiles de abordar, que incremente los recursos de apoyo.

Pero, en este caso, estamos referidos a un proceso de construcción permanente, individual y colectivo, de producción intersubjetiva de relaciones sociales de retención y reconstrucción del pasado, de aceptación de identidades y diferencias, de presente y futuro, de hacer solidaridad hasta formar un cuerpo en el mundo social. La red será tanto más promotora de aspectos pro-sociales, en cuanto posee por sí misma identidad, cohesión y las habilidades para encontrar y expresar sus propios recursos.

Es entonces cuando genera soluciones creativas, transformadoras de cara a situaciones dificiles. Efectivamente tenemos redes como estas en el pais, eso se puede constatar, pero en la mayoria de los casos y desgraciadamente la acción "interventiva" en redes, se ha entendido como una propuesta, predominantemente estatal, organizada en torno a la refrendación de la asistencialidad, tratando de integrar y más bien privilegiar la visión institucional con la comunitaria y lo reticular. El concepto de red usado está investido como forma de coordinación de espacios institucionales que más se acomodan a los programas financiados por el Estado, que a la creación de espacios de desarrolio de diferentes y nuevas alternativas de acción, de posibilidades de activación de conductas grupales, de articulación. de perspectivas de los grupos sociales implicados, de movilización de solidaridad, del afecto positivo y el compromiso. Las redes nacen de lo que se está haciendo o siendo y no desde 10 que se les trae, como algo que llega a un vacío, que va a ser llenado. No hay tal vacío, las agrupaciones y redes ya están, existen antes. No se ha sabido entender y aceptar este aspecto y reconocer los contextos reales.

El modelo de trabajo de red dominante involucra más los intercambios que los vinculos, la dependencia que el apoyo y responsabilidad. La acción interventiva se genera desde la noción de práctica reticular donde los sujetos institucionales determinan al sujeto foco, que termina siendo el eterno deshabilitado en déficit y lleno de carencias, que será intervenido desde mediadores sociales que propician sistemas de intercambio y distribución.

En resumen, si no se atiende a una reflexión seria se puede incluso producir un nuevo fenómeno de repiiegue. La intervención debe tener sentido estructural y transversal a los problemas económicos, al reconocimiento de la subjetividad $y$ a la libertad en la constitución de los limites. No puede ser dispersa y debe operar sobre redes reales.

No hay, en esta perspectiva, ningún recetario magistrai ni para "formarlas" ni para esperar que sólo desde ellas se pueda superar el agravio a lo humano que supone el peso de un modelo económico sobre la sociedad civil. Vale más el esfuerzo de aceptar que hay agrupamientos que son reales (barras de fútbol, rock juvenil urbano, etc.) y que se constituyen desde ellos como verdaderas formas de resistencia en pos de la participación y del control intentando, desde sus identidades, conseguir superar fragmentaciones, reconocerse y aglutinar. Sin duda, a veces, su discurso o propuesta "no nos simpatizan", pero ellos están allí. Cada dia refuerzan su presencia, existencia y quehacer.

Desde alli pueden surgir agentes y posiciones de transformación que satisfagan las necesidades y problemas de salud y desarrollo de la gente. 
Para elio es indispensable una firme voluntad de cambio en los hacedores de políticas, una más generosa y mejor distribuida asignación de presupuestos y un marco cultural y político en que el bienestar social y la calidad subjetiva de vida de los ciudadanos debe ser prioritario y tener una racionalidad de integración con plenos derechos.

Es evidente que en salud mental y, particularmente, en el campo de los "problemas ppsicosociales" se hace necesario un sistema de apoyo y de relación comunitaria. Esto aclara la creciente tendencia a trabajos desde este nivel y enfoque. No sólo en el tema denominado drogodependencias, sino en otros como violencia doméstica, maltrato infantil, etc. Se trata así de crear un patrón duradero de lazos continuos que jueguen un rol significativo en el mantenimiento de la integridad psicológica de las personas. En general, los análisis evaluativos informan de tres situaciones que se ven afectadas positivamente: la recuperación de la persona, el rol social y el apoyo socio-emocional.

Sin embargo, hay otros antecedentes que es necesario tomar en cuenta, por su impacto en la efectividad y en el sentido del proceso que se quiere "instalar". Uno es la situación socioeconómica de la comunidad, donde claramente se establece que las penurias y tensiones generadas por la pobreza y la injusticia social, disminuye severamente las bondades del apoyo que pueden ofrecer las relaciones en el vecindario. Esto empeora cuando los servicios sociales son insuficientes o inadecuados. Las comunidades de mayor tamaño se involucran menos, puesto que predominan patrones conductuales que reducen la interacción y la participación y que se hacen muy evidentes cuando hay una historia de fragmentación social, desaliento y frustración.

Estos y otros aspectos han sido pasados por alto y deben ser corregidos drásticamente. Es urgente revisar lo que se hace y reflexionar con seriedad puesto que este podría ser otro proceso desafortunado que producto de visiones tecnologizadas y reduccionistas, produce un nuevo efecto de impotencia, rabia y desesperanza en trabajadores sociales, educadores y vecinos.

No se puede castrar un tipo de acción social transformadora y humanizadora por limitaciones como el no considerar que el desempleo y la inequidad influyen en el desarrollo del malestar psicológico y sindromes conductuales y psicopatológicos.

Este tipo de acciones, ha sido presentada en Chile como la nueva promesa de reorganización social, en ella estaria la verdad y la solución de todos nuestros problemas, produciéndose así una arremetida irrefrenable y verticalista, instalada en capacitaciones, documentos, video-grabadores, altoparlantes y autoadhesivos. La novedad del año: jhay que agruparsel. Red pasa a ser la contraseña del lenguaje. Las puertas de los fondos y proyectos se abrirán de par en par para los más ágiles.

En resumen, la gestión de intervención psicosocial en redes ha operado, como una acción básicamente estatal, local, con alta difusión programática, vertical y autoritaria, funcionando en los espacios institucionales que más acomodan a los programas financiados por el verticalismo centralista. Se ha concebido a la red desde una doble situación: como sistemas de conversaciones y como sistemas de acción. Pero no se ha considerado relevante que las redes y agrupamientos ya existen, por ejemplo, las juveniles de origen espontáneo, autocontenidas, autorreferidas, con un hacer colectivo centrado en "sus" experiencias, incluso frente a la participación institucional (11). Esta y otras redes naturales integran y desarrollan una conciencia de la posición de exclusión social, produciendo expresiones de identidad, culturales y de estilos de intercambio y recreación. En el tema "consumos", no pocas veces, desde ambientes deprivados y alejados del poder formal, estos agrupamientos establecen alianzas con la red del mejor tipo de micro-empresa familiar que ha producido el modelo de desarrollo: el micro-tráfico de substancias ilegales.

En los programas hay un abandono de las dimensiones culturales y sociales, del soporte de las personas proveedoras y participativos (por ejemplo, mujeres y madres de vocación comunitaria), y de la necesidad de enfrentar la relación entre tensión y apoyo.

En definitiva, la principal constatación que se puede hacer de los programas de prevención en red es obvia, pero desafortunadamente no fue considerada desde los sectores académicos y gubernamentales; las comunidades no emergen, ni se construyen como efecto de la intervención. Eso es negar, de nuevo, la participación.

Creemos que un aspecto clave que corresponde a la psicologia social, es transcender y cuestionar sus propias posibilidades aparentemente sólo técnicas y analizar los valores y metas macro y microsociales con la capacidad de los individuos y grupos, históricamente construida, para generar satisfacciones a las necesidades sociopsicológicas y debatir cómo y en qué medida estas dimensiones se ven afectadas por los sucesos, transformaciones y procesos macrosociales.

Revisar este potencial aporte de la psicología social, puede contribuir al análisis integral de los problemas sociales y de los programas de acción social, pero implica coger el tren de la historia y desgranar con rigor los soportes teóricos y metodológicos: conceptos como intención de conducta, autoestima, prejuicios, actitudes, cooperación, estrés psicosocial, representaciones, deben re-plantearse no sólo desde las teorías que les dan contenido, sino que también desde las distintas coyunturas socio-políticas en las que aparecen abasteciendo los programas de acción social. Esta vía es necesaria relevarla para poder avanzar por los dificiles caminos de la intervención psicosocial.

\section{Bibliografía}

1. ALFARO, J. (1999), Psicología social e intervención, Ediciones Fondo de Desarrollo de la Docencia, Universidad Diego Portales, Santiago, Chile.

2. ASUN, D. et. al. (1992), Psicologia comunitaria y salud mental en Chile, Universidad Diego Portales, Santiago, Chile.

3. ASUN, D., PAEZ, D. y VERA, A. (1999), Análisis de los fundamentos, modelos y propuestas de la investigación psicosocial en Chile (1975- 
1996), Programa colectivo de estudio psicosocial, Universidad Diego Portales, Santiago, Chile.

4. MINSAL. (1999), Las enfermedades mentales en Chile. Magnitud y consecuencias, Unidad de Salud mental.

5. ARENSBURG, S. y COVACEVICH, C. (1997), El poder de las palabras: Articulación psicología-drogas, Tesis de psicología, Universidad Diego Portales, Santiago, Chile.

6. JIMENEZ, F. y BURILLO, F. (1997), Psicologia social y sistema penal, Alianza Universidad, Madrid, España.

7. CHALAIYE, S. (1997), Agresores sexuales intrafamiliares, Tesis de Psicologia, Universidad Diego Portales, Santiago, Chile.

8. MAGENDZO, S. y TOLEDO, M. l. (1990), Soledad y deserción: Un estudio psicosocial de la deserción escolar en los sectores populares, P.I.E.E., Chile.
9. ASUN, D., VERA, A. y WOOD, P. (1994), Un modelo explicativo de la salud mental basado en categorias psicosociales; Descripción teórica de sus componentes, construcción de una bateria de instrumentos para la evaluación de factores psicosociales y prueba empirica del modelo, Escuela de Psicologia, Universidad Diego Portales, Santiago, Chile.

10. DOCUMENTO "Psicología social: pertinencia, retos y desafios", en Encuentro Metropolitanos de estudiantes de Psicologia social, Universidad de Chile, ARCIS, Universidad Diego Portales, etc.

11. SALAZAA, G. (1998), Drogas y comunidad local. Economia, sociedad, politica. El caso de la comuna El Bosque (1982-1997), Sur Profesionales, Santiago, Chile. 